\title{
Role of betaine in improving the antidepressant effect of S-adenosyl-methionine in patients with mild-to-moderate depression
}

This article was published in the following Dove Press journal:

Journal of Multidisciplinary Healthcare

16 January 2015

Number of times this article has been viewed

\author{
Francesco Di Pierro' \\ Rossana Orsi² \\ Roberto Settembre ${ }^{3}$ \\ 'Scientific Department, Velleja \\ Research, Milan, Italy; ${ }^{2} \mathrm{ASL}$ TOI, Turin, \\ Italy; ${ }^{3}$ Neurosurgery Department, ASL \\ BA, Di Venere Hospital, Bari, Italy
}

Background: $S$-adenosyl-methionine (SAMe) is a common add-on treatment used to counteract depressive symptoms in subjects with mild-to-moderate depression, who are low responders to other antidepressant drugs. However, there is some concern about the possible impact of SAMe therapy on homocysteine levels. Betaine is known both to counteract high level of homocysteine in plasma and to increase liquor and plasma levels of SAMe, thus potentiating its effect.

Aim: To evaluate the role played by betaine, administered along with $\mathrm{SAMe}$, in potentiating the antidepressive role played by SAMe administered as such.

Methods: The study enrolled 46 subjects with a diagnosis of mild-to-moderate depression according to the Beck Depression Inventory Scale II. All the subjects had a suboptimal control of their symptoms. After randomization, they were treated with adjunctive treatment with either Samyr ${ }^{\circledR}$ (enteric-coated SAMe) or DDM Metile ${ }^{\circledR}$ (enteric-coated SAMe plus betaine) for 90 days.

Results: Both treatments acted similarly in improving symptoms such as anxiety, psychomotor agitation, feelings of helplessness and worthlessness, physical efficiency, and somatization, but treatment with DDM Metile ${ }^{\circledR}$ determined better statistically significant results following a 90-day therapy. Tolerability and compliance were overlapping in both the treatments.

Conclusion: The association of SAMe plus betaine seemed to demonstrate more effectiveness than SAMe alone when administered as an add-on therapy to subjects, affected by mild-tomoderate depression, who were low responders to conventional antidepressants.

Keywords: DDM Metile ${ }^{\circledR}$, Samyr ${ }^{\circledR}$, Betaine, SAMe, mild-to-moderate depression, methyl donor

\section{Introduction}

$S$-adenosyl-methionine (SAMe) is a methyl donor that is most commonly used in the treatment of mild-to-moderate depression and osteoarthritis and as a liver-protective agent. ${ }^{1}$ Although its use in serious depression is still controversial, ${ }^{2,3}$ its involvement in the synthesis of such neurotransmitters as norepinephrine, serotonin, and dopamine has been ascertained. ${ }^{4}$ In cells, SAMe is synthesized starting from amino acid L-methionine through a metabolic pathway, known as "one-carbon cycle", whose proper performance requires an adequate quantity of folate and vitamin B12. ${ }^{5}$ Folate and vitamin B12, in turn, are coenzymes of a large number of metabolic pathways, including the so-called "homocysteine cycle", where they work together with vitamin B6 and betaine to reduce blood homocysteine level. ${ }^{6}$ If the latter rises, it may increase the depressive event as a consequence of the subtraction of factors required by SAMe synthesis. ${ }^{7,8}$ In fact, it has been ascertained that the administration of folate (just by way of example) can reduce
Correspondence: Francesco Di Pierro Scientific Department, Velleja Research, Viale Lunigiana 23, Milan 20125, Italy Tel +393495527663

Fax +390523511894

Email f.dipierro@vellejaresearch.com 
the depressive syndrome or enhance the pharmacological action exerted by other antidepressants. ${ }^{9,10}$ On the other hand, the oral administration of SAMe may involve a potential risk of increased blood homocysteine level, ${ }^{11}$ as homocysteine may detract folate and vitamin B12 from the biochemical pathway of endogenous synthesis of SAMe, thus reducing the temporal antidepressive effects of the latter. It is also well known that oral administration of betaine - a methyl donor participating exclusively in the homocysteine cyclecauses both homocysteine reduction and increased blood SAMe level. ${ }^{12}$ Therefore, by considering 1) B-group vitamin detraction, consequent to hyperhomocysteinemia conditions, may induce a deficit in SAMe synthesis; 2) administration of SAMe may result in hyperhomocysteinemia; and 3) administration of betaine both reduces the homocysteine level in blood and increases SAMe concentration, we decided to verify whether the combined action of SAMe and betaine in patients with mild-to-moderate depression would prove to be more effective than the administration of SAMe alone.

\section{Materials and methods Study design}

This 3-month, randomized, observational, controlled trial was conducted in the setting of routine practice, in accordance with the principles stated in the Declaration of Helsinki and consistent with Good Clinical Practice, as defined by the International Conference on Harmonization and in accordance with the ethical principles underlying European Union Directive 2001/20/EC and the United States Code of Federal Regulations, Title 21, Part 50 (21CFR50). ${ }^{13}$ The protocol, subjects' consent, and privacy forms were approved by the local review board. All patients provided their written informed consent to participate in this study after a full explanation of the study had been given. The study was conducted in a single center in Italy (Turin) between May 2011 and May 2012 on 46 patients enrolled on the basis of the diagnosis of mild-to-moderate depression (score between 14 and 28) as evaluated according to the Beck Depression Inventory Scale II. ${ }^{14}$ Forty-five of the 46 patients completed the study and were evaluated from a statistical point of view.

\section{Inclusion criteria}

Inclusion criteria were the following: age between 18 years and 75 years; participants' signing of the informed consent; diagnosis of mild-to-moderate depression; and patients having been treated with antidepressive drugs (excluding SAMe-based agents) for at least 6 months with suboptimal control of symptoms.

\section{Exclusion criteria}

Exclusion criteria were the following: diagnosis of serious depression, psychosis, or bipolar disorder; abuse of alcohol or drugs; suicidal ideation; neurological diseases; epilepsy; pregnancy; breastfeeding; and use of oral or intravenous SAMe in the previous 3 months.

\section{Study protocol}

The study scheme is presented in Figure 1. After being enrolled, all the subjects started taking, as adjunctive therapy, either SAMe or SAMe plus betaine for the whole length of the study (90 days). Both treatments were administered on an empty stomach. Medical checks, aimed to evaluate the symptoms trend, were performed from $T=0$ to $T=90$ every 15 days.

\section{Tested products}

Patients were treated with an adjunctive therapy with either $400 \mathrm{mg}$ of SAMe formulated as enteric-coated tablets $\left(\right.$ Samyr $^{\circledR}$, Abbott Laboratories, USA) or an enteric-coated formulation (DDM Metile ${ }^{\circledR}$, Omeopiacenza, Italy) containing $250 \mathrm{mg}$ of SAMe (Gnosis, Italy) plus $125 \mathrm{mg}$ of betaine per tablet (Procemsa, Italy). Samyr ${ }^{\circledR}$ was administered twice a day every 12 hours. DDM Metile ${ }^{\circledR}$ was administered three times a day every 8 hours. Samyr ${ }^{\circledR}$ is a nonreimbursable drug (C-type), with the following marketing authorization

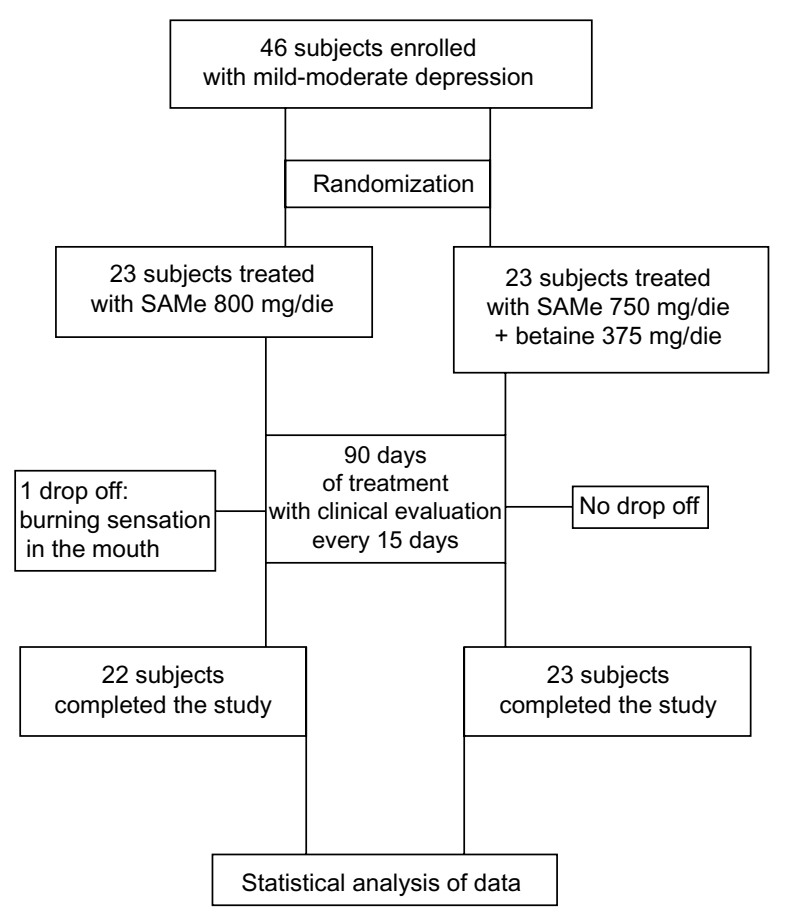

Figure I Scheme of the study.

Abbreviation: SAMe, S-adenosyl-methionine. 
number: 022865188 . DDM Metile ${ }^{\circledR}$, in agreement with Italian law, was registered with the Ministry of Health in 2010 (registration number: 52171) as a food supplement. Both actives (SAMe and betaine) of DDM Metile ${ }^{\circledR}$ are accepted ingredients, as far as quality and quantity are concerned, for nutraceutical formula; all excipients used to formulate DDM Metile $^{\circledR}$ are also food-grade ingredients.

\section{Concomitant antidepressive drugs}

Due to the randomization procedure in the Samyr ${ }^{\mathbb{B}}$ group, eight subjects were under treatment with one antidepressant; nine subjects were under treatment with one antidepressant plus one benzodiazepine; three subjects were under treatment with two antidepressants; and three subjects were under treatment with two antidepressants plus one benzodiazepine. In detail, six of the 23 subjects were under treatment with bupropion $(150 \mathrm{mg} / \mathrm{die})$; one with bupropion $(150 \mathrm{mg} / \mathrm{die})$ and escitalopram (10 mg/die); three with bupropion (150 mg/die), bromazepam (3 mg/die), and mirtazapine (15 mg/die); four with paroxetine $(20 \mathrm{mg} / \mathrm{die})$ and etizolam $(1 \mathrm{mg} / \mathrm{die})$; two with citalopram (20 mg/die); three with alzopram (3 mg/die) and sertraline (50 mg/die); two with etizolam ( $1 \mathrm{mg} / \mathrm{die})$ and duloxetine $(60 \mathrm{mg} / \mathrm{die})$; and two with paroxetine $(20 \mathrm{mg} / \mathrm{die})$ and trazodone $(50 \mathrm{mg} / \mathrm{die})$. In the DDM Metile ${ }^{\circledR}$ group, 13 subjects were under treatment with one antidepressant; six subjects were under treatment with one antidepressant plus one benzodiazepine; two subjects were under treatment with two antidepressants; and two subjects were under treatment with two antidepressants plus one benzodiazepine. In detail, four of 23 patients were under treatment with bupropion (150 mg/die); two with bupropion (150 mg/die) and escitalopram (10 mg/die); two with bupropion (150 mg/die), bromazepam (3 mg/die), and mirtazapine (15 mg/die); six with paroxetine $(20 \mathrm{mg} / \mathrm{die})$; three with paroxetine $(20 \mathrm{mg} / \mathrm{die})$ and etizolam ( $1 \mathrm{mg} / \mathrm{die})$; three with citalopram (20 mg/die); two with alzopram ( $3 \mathrm{mg} / \mathrm{die})$ and sertraline (50 mg/die); and one with etizolam $(1 \mathrm{mg} / \mathrm{die})$ and duloxetine $(60 \mathrm{mg} / \mathrm{die})$. After a 30-day treatment with the add-on therapy, one subject of the SAMe group under treatment with bupropion and escitalopram opted out due to unwanted effects (burning sensation in the mouth).

\section{Endpoints}

The primary efficacy endpoints of the study were assessed through subjective global symptoms relief (anxiety, psychomotor agitation, feelings of helplessness and worthlessness, physical efficiency, and somatization) according to an adapted version of the visual-analog scale (0-10) developed by Huskisson et al. ${ }^{15}$ The secondary endpoints included the occurrence of side effects, tolerability, and compliance. Tolerability and compliance were scored as very poor, poor, fairly good, good, and excellent. Primary and secondary endpoints were evaluated every 15 days after enrolment.

\section{Statistical analysis}

The Wilcoxon signed rank test was used to analyze any differences from baseline scores inside the same group of treatment, while the Mann-Whitney score rank test was used to analyze any score differences between the two groups. Values were considered significant at $P<0.05$.

\section{Results}

A total of 46 subjects with a diagnosis of mild-to-moderate depression were enrolled in the trial. Forty-five of these completed the study. A total of 23 were randomized to receive SAMe and 23 were randomized to receive SAMe plus betaine both as add-on therapy to the previously prescribed antidepressive drugs. As mentioned in the Materials and Methods section, once the enrolment and randomization procedures had been completed, the individuals started their oral 90-day treatment with either SAMe or SAMe plus betaine tablets. The characteristics of the randomized groups are shown in Table 1. The results of the treatments are shown in Tables 2-6. As can be seen, at $T=0$, all the baseline parameters did not show any statistically significant difference between the two groups. In addition, both treatments, SAMe and SAMe plus betaine, demonstrated to be effective in improving the subjective parameters analyzed - anxiety, psychomotor agitation, feelings of helplessness and worthlessness, physical efficiency, and somatization. However, the statistical analysis performed to establish a direct comparison between the two treatments demonstrated a better performance of the SAMe-plus-betaine therapy versus the one resulting from SAMe alone. As a matter of fact, at $T=90$ all parameters of the SAMe/betaine group showed a significant difference in comparison with the same parameters of the SAMe treatment. In addition, treatment with SAMe plus betaine seemed to

Table I Features of participants on enrolment

\begin{tabular}{lll}
\hline & SAMe $(\mathbf{N}=23)$ & SAMe/betaine $(\mathbf{N}=\mathbf{2 3})$ \\
\hline Sex (males/females) & $6 / 17$ & $7 / 15$ \\
Female age (years) & $52.8 \pm 9.4$ & $53.4 \pm 11.8$ \\
Male age (years) & $50.3 \pm 10.3$ & $51.4 \pm 7.6$ \\
Months from diagnosis & $18.6 \pm 5.2$ & $20.3 \pm 6.6$ \\
\hline
\end{tabular}

Note: All values are expressed as median \pm standard deviation. Abbreviation: SAMe, S-adenosyl-methionine. 
Table 2 Evaluation of the parameter "anxiety"

\begin{tabular}{llllll}
\hline Time, & SAMe & $\boldsymbol{P}$ & SAMel & $\boldsymbol{P}$ & $\boldsymbol{P}$ \\
$\boldsymbol{T}$ (days) & (A) & (vs T=0) & betaine (B) & (vs T=0) & (B vs A) \\
\hline 0 & $7.00 \pm 1.24$ & & $7.18 \pm 1.14$ & & $\mathrm{~ns}$ \\
15 & $5.78 \pm 1.28$ & $<0.05$ & $4.95 \pm 1.33$ & $<0.05$ & $\mathrm{~ns}$ \\
30 & $4.87 \pm 1.22$ & $<0.05$ & $4.32 \pm 1.32$ & $<0.01$ & $\mathrm{~ns}$ \\
45 & $4.04 \pm 1.26$ & $<0.01$ & $3.55 \pm 1.47$ & $<0.01$ & $\mathrm{~ns}$ \\
60 & $3.52 \pm 1.04$ & $<0.01$ & $2.95 \pm 1.36$ & $<0.01$ & $\mathrm{~ns}$ \\
75 & $3.09 \pm 1.31$ & $<0.01$ & $2.23 \pm 1.23$ & $<0.01$ & $\mathrm{~ns}$ \\
90 & $2.39 \pm 1.34$ & $<0.01$ & $1.23 \pm 1.15$ & $<0.01$ & $<0.05$ \\
\hline
\end{tabular}

Note: All values are expressed as median \pm standard deviation.

Abbreviations: ns, not significant; SAMe, S-adenosyl-methionine.

Table 3 Evaluation of the parameter "psychomotor agitation"

\begin{tabular}{llllll}
\hline Time, & SAMe & $\boldsymbol{P}$ & SAMel & $\boldsymbol{P}$ & $\boldsymbol{P}$ \\
$\boldsymbol{T}$ (days) & (A) & (vs T=0) & $\begin{array}{l}\text { betaine (B) } \\
\text { (vs T=0) }\end{array}$ & (B vs A) \\
\hline 0 & $4.39 \pm 1.73$ & & $4.27 \pm 1.70$ & & $\mathrm{~ns}$ \\
15 & $3.74 \pm 1.76$ & $<0.05$ & $2.86 \pm 1.64$ & $<0.05$ & $\mathrm{~ns}$ \\
30 & $2.78 \pm 1.59$ & $<0.01$ & $2.18 \pm 1.50$ & $<0.01$ & $\mathrm{~ns}$ \\
45 & $2.30 \pm 1.46$ & $<0.01$ & $2.09 \pm 1.72$ & $<0.01$ & $\mathrm{~ns}$ \\
60 & $2.09 \pm 1.12$ & $<0.01$ & $1.45 \pm 1.06$ & $<0.01$ & $\mathrm{~ns}$ \\
75 & $2.09 \pm 0.90$ & $<0.01$ & $1.32 \pm 1.04$ & $<0.01$ & $\mathrm{~ns}$ \\
90 & $1.57 \pm 0.66$ & $<0.01$ & $0.95 \pm 0.65$ & $<0.01$ & $<0.05$ \\
\hline
\end{tabular}

Note: All values are expressed as median \pm standard deviation.

Abbreviations: ns, not significant; SAMe, S-adenosyl-methionine.

Table 4 Evaluation of the parameter "feelings of helplessness and worthlessness"

\begin{tabular}{llllll}
\hline $\begin{array}{l}\text { Time, } \\
\boldsymbol{T} \text { (days) }\end{array}$ & $\begin{array}{l}\text { SAMe } \\
\text { (A) }\end{array}$ & $\begin{array}{l}\boldsymbol{P} \\
\text { (vs T=0) }\end{array}$ & $\begin{array}{l}\text { SAMel } \\
\text { betaine (B) }\end{array}$ & $\begin{array}{l}\boldsymbol{P} \\
\text { (vs } \boldsymbol{T}=\mathbf{0})\end{array}$ & $\begin{array}{l}\boldsymbol{P} \\
\text { (B vs A) }\end{array}$ \\
\hline 0 & $6.00 \pm 1.45$ & & $6.09 \pm 1.66$ & & $\mathrm{~ns}$ \\
15 & $4.83 \pm 1.19$ & $<0.05$ & $4.55 \pm 1.06$ & $<0.01$ & $\mathrm{~ns}$ \\
30 & $4.17 \pm 1.27$ & $<0.01$ & $4.09 \pm 1.19$ & $<0.01$ & $\mathrm{~ns}$ \\
45 & $3.39 \pm 1.31$ & $<0.01$ & $2.77 \pm 1.54$ & $<0.01$ & $\mathrm{~ns}$ \\
60 & $2.78 \pm 0.95$ & $<0.01$ & $2.18 \pm 1.33$ & $<0.01$ & $\mathrm{~ns}$ \\
75 & $2.30 \pm 0.82$ & $<0.01$ & $1.77 \pm 1.11$ & $<0.01$ & $\mathrm{~ns}$ \\
90 & $2.13 \pm 0.63$ & $<0.01$ & $1.18 \pm 1.01$ & $<0.01$ & $<0.01$ \\
\hline
\end{tabular}

Note: All values are expressed as median \pm standard deviation.

Abbreviations: ns, not significant; SAMe, S-adenosyl-methionine.

Table 5 Evaluation of the parameter "physical efficiency"

\begin{tabular}{|c|c|c|c|c|c|}
\hline $\begin{array}{l}\text { Time, } \\
T \text { (days) }\end{array}$ & $\begin{array}{l}\text { SAMe } \\
\text { (A) }\end{array}$ & $\begin{array}{l}P \\
(\text { vs } T=0)\end{array}$ & $\begin{array}{l}\text { SAMel } \\
\text { betaine (B) }\end{array}$ & $\begin{array}{l}P \\
(\text { vs } T=0)\end{array}$ & $\begin{array}{l}P \\
\text { (B vs A) }\end{array}$ \\
\hline 0 & $2.26 \pm 1.21$ & & $2.27 \pm 1.24$ & & ns \\
\hline 15 & $3.96 \pm 1.22$ & $<0.05$ & $4.00 \pm 1.23$ & $<0.05$ & ns \\
\hline 30 & $4.52 \pm 1.31$ & $<0.01$ & $4.82 \pm 1.59$ & $<0.01$ & ns \\
\hline 45 & $5.09 \pm 1.28$ & $<0.01$ & $5.55 \pm 1.50$ & $<0.01$ & ns \\
\hline 60 & $5.70 \pm 0.82$ & $<0.01$ & $6.27 \pm 1.12$ & $<0.01$ & ns \\
\hline 75 & $5.52 \pm 1.04$ & $<0.01$ & $6.23 \pm 1.45$ & $<0.01$ & ns \\
\hline 90 & $5.70 \pm 0.88$ & $<0.01$ & $6.68 \pm 1.17$ & $<0.01$ & $<0.05$ \\
\hline
\end{tabular}

Note: All values are expressed as median \pm standard deviation.

Abbreviations: ns, not significant; SAMe, S-adenosyl-methionine.
Table 6 Evaluation of the parameter "somatization"

\begin{tabular}{|c|c|c|c|c|c|}
\hline $\begin{array}{l}\text { Time, } \\
T \text { (days) }\end{array}$ & $\begin{array}{l}\text { SAMe } \\
\text { (A) }\end{array}$ & $\begin{array}{l}P \\
\text { (vs } T=0)\end{array}$ & $\begin{array}{l}\text { SAMel } \\
\text { betaine (B) }\end{array}$ & $\begin{array}{l}P \\
\text { (vs } T=0)\end{array}$ & $\begin{array}{l}P \\
\text { (B vs A) }\end{array}$ \\
\hline 0 & $6.96 \pm 0.77$ & & $7.27 \pm 1.16$ & & ns \\
\hline 15 & $4.61 \pm 1.27$ & $<0.05$ & $4.50 \pm 1.19$ & $<0.05$ & ns \\
\hline 30 & $3.91 \pm 1.16$ & $<0.01$ & $3.82 \pm 1.11$ & $<0.01$ & ns \\
\hline 45 & $3.17 \pm 0.94$ & $<0.01$ & $3.05 \pm 0.95$ & $<0.01$ & ns \\
\hline 60 & $2.83 \pm 0.72$ & $<0.01$ & $2.27 \pm 1.17$ & $<0.01$ & ns \\
\hline 75 & $2.26 \pm 0.72$ & $<0.01$ & $1.45 \pm 1.34$ & $<0.01$ & ns \\
\hline 90 & $2.09 \pm 0.79$ & $<0.01$ & $0.55 \pm 0.80$ & $<0.01$ & $<0.01$ \\
\hline
\end{tabular}

Note: All values are expressed as median \pm standard deviation.

Abbreviations: ns, not significant; SAMe, S-adenosyl-methionine.

yield better results even considering the symptoms trend from $T=15$ to $T=75$. In fact, even if really significant results do not seem to occur, the results show a tendency where the SAMe-plus-betaine treatment improves symptoms in a slightly better way when compared with the SAMe group. With regard to the symptom "anxiety", treatment with SAMe plus betaine, compared with the SAMe therapy, improves the score by about $10 \%$ for each point considered and by about $20 \%$ at $T=90$; symptom "psychomotor agitation" improved by about $15 \%$ for each point considered; symptom "feelings of helplessness and worthlessness" improved by about $10 \%$ for each point considered and by about $15 \%$ at $T=90$; symptom "physical efficiency" improved by about $17 \%$, by average, and by about $40 \%$ at $T=90$; and finally, the symptom "somatization" improved by about $8 \%$, by average, and by about $18 \%$ at $T=90$ (Figures 2 and 3). With regard to tolerability (Table 7), both treatments seemed to be well tolerated; one (female) subject of the SAMe group did not complete the study, the reason for her early withdrawal (occurred at $T=30$ ) being excessive burning sensation in the mouth. In terms of compliance, the two treatments resulted in overlap (Table 8).

\section{Discussion}

In the clinical practice aimed at the treatment of depression, SAMe is commonly combined with a drug of a different class - for example, a serotonin reuptake inhibitor or a tricyclic antidepressant - to improve the clinical results of the latter and/or increase the rate of individuals responding to the therapy provided and/or improve the patients' cognitive function, whose deterioration often accompanies depressive syndrome. ${ }^{16}$ From the biochemical point of view, however, administration of SAMe could, at least theoretically, increase the cardiovascular health risk in these patients because of a higher blood homocysteine level resulting from 


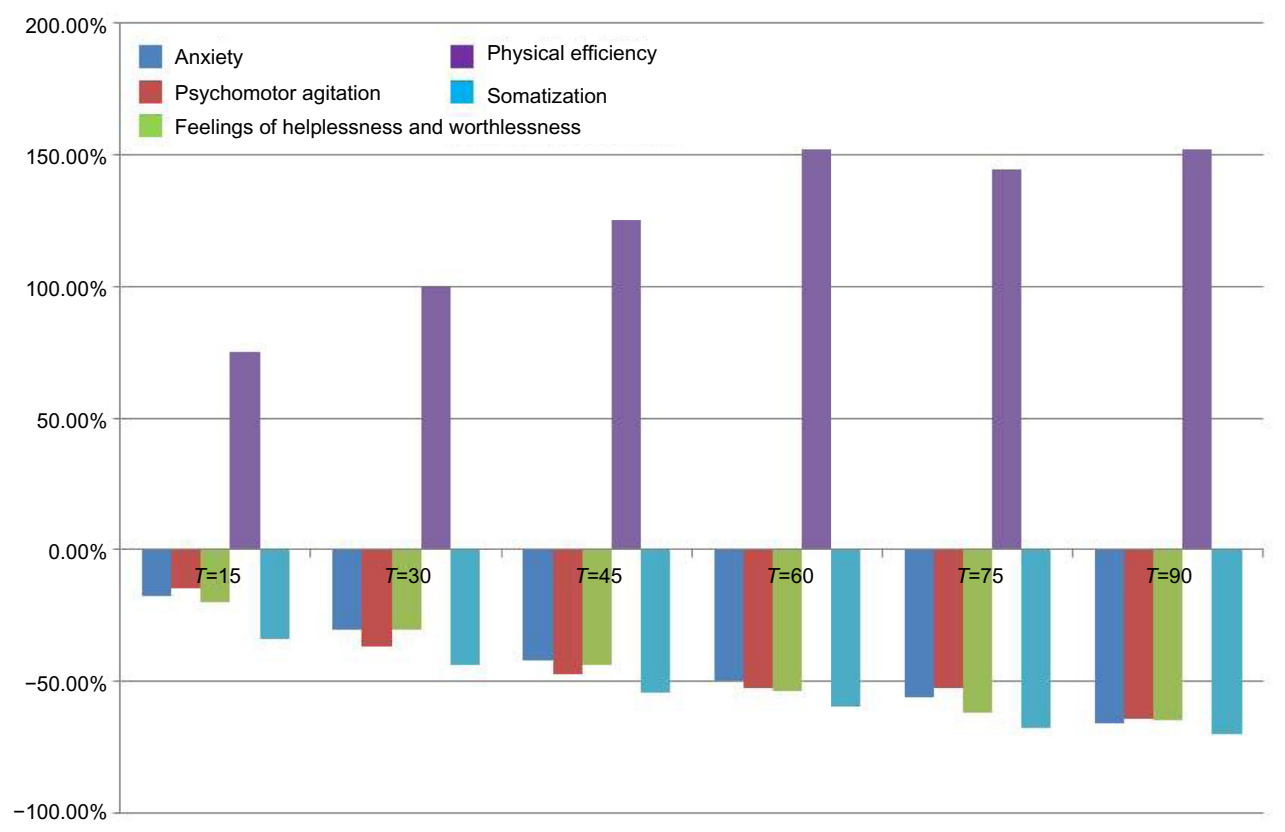

Figure 2 Score percentage variation versus $T=0$ in subjects treated with $S$-adenosyl-methionine (SAMe).

SAMe metabolism. Today we know that administration of folate, vitamin B6, vitamin B12, and/or betaine (also referred to as trimethylglycine) can counteract the hyperhomocysteinemia condition. ${ }^{17-20}$ In addition, the elderly patient may be facing a SAMe deficit in blood as a consequence of increased blood homocysteine levels. ${ }^{7,8}$ These two factors, SAMe and homocysteine, seem to be firmly connected, and betaine could be one of the possible links between them.
It is indeed known that in the elderly patient, administration of betaine increases endogenous SAMe concentration. ${ }^{12}$ However, a recent trial ${ }^{21}$ demonstrated that despite the concerns about the impact that a SAMe therapy may have on homocysteine levels and the risk of adverse cardiovascular effects, the lack of any significant increase in total homocysteine levels following treatment suggests that no toxic effects should be expected from SAMe. Such a finding, however,

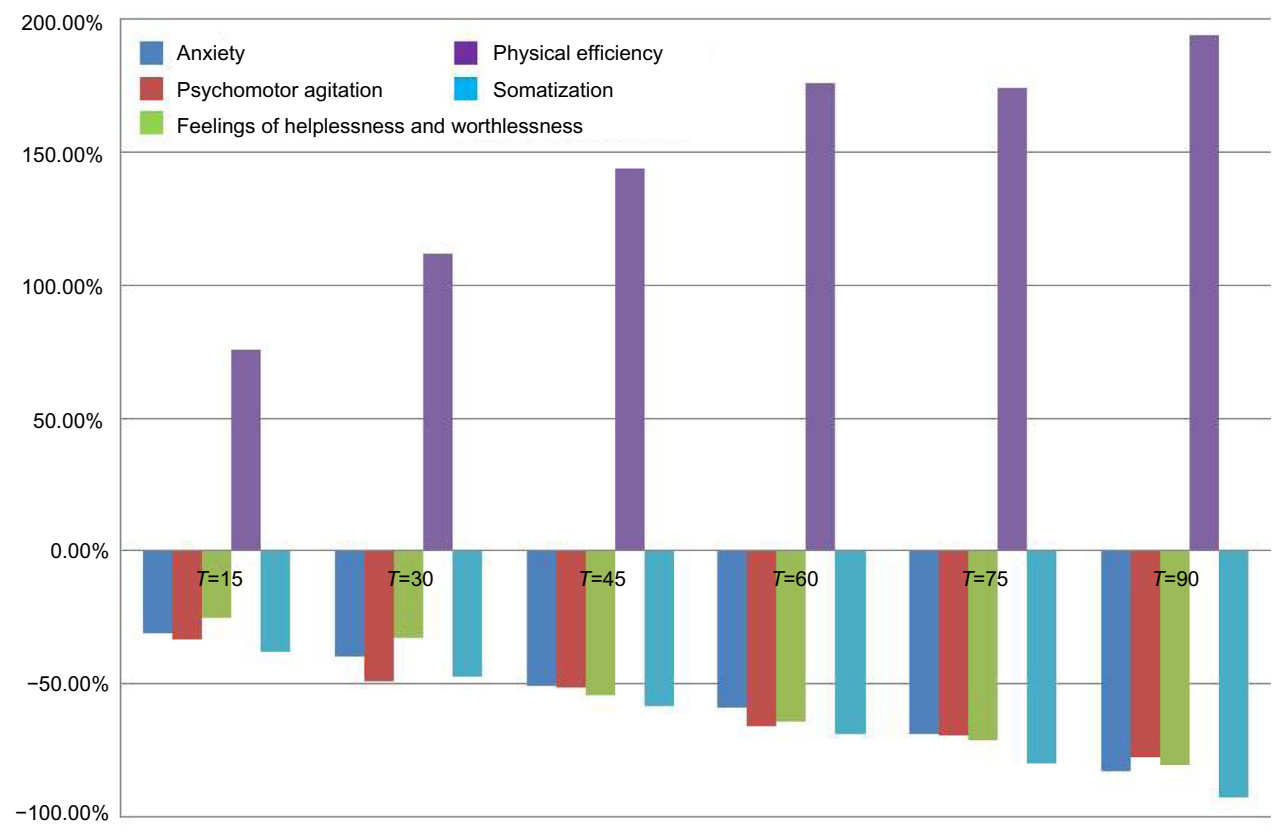

Figure 3 Score percentage variation versus $T=0$ in subjects treated with $S$-adenosyl-methionine (SAMe)/betaine. 
Table 7 Tolerability assessment

\begin{tabular}{llll}
\hline Tolerability & N (SAMe) & N (SAMe/betaine) & $\boldsymbol{P}$ \\
\hline Poor & $\mathrm{I}$ & 0 & $\mathrm{~ns}$ \\
Fair & 2 & 2 & $\mathrm{~ns}$ \\
Good & 3 & $\mathrm{I}$ & $\mathrm{ns}$ \\
Excellent & 17 & 20 & $\mathrm{~ns}$ \\
\hline
\end{tabular}

Abbreviations: $\mathrm{ns}$, not significant; SAMe, S-adenosyl-methionine.

has some significant limitations and should be interpreted with caution. As a matter of fact, SAMe administration does raise the levels of plasma $S$-adenosyl homocysteine. ${ }^{21}$ Starting from the assumption that administration of SAMe may reduce depressive symptoms on the one hand and remove endogenous SAMe on the other, thus counteracting, with time, the benefits resulting from this administration, we decided to assess the impact of an adjunctive therapy with SAMe, regardless of whether it was combined with betaine, in individuals suffering from mild-to-moderate depression and apparently poorly responsive to an antidepressant-based treatment. As a matter of fact, the preliminary results of this assessment seem to point to a major role played by betaine in improving the symptoms of individuals suffering from mild-to-moderate depression. The possible explanation of this result is not simple, but some assumptions can be made. The administration of betaine may reduce plasma homocysteine concentrations, which may result, for example, in folate saving, with the latter substance being made available to increase endogenous SAMe synthesis. Unfortunately, this hypothesis cannot be confirmed as the protocol of this study did not provide for plasma collection to assess plasma homocysteine or folate concentration. On the other hand, betaine may also directly counteract the accumulation of $S$-adenosyl homocysteine in plasma while increasing the SAMe rate. Even in this case, this assumption cannot be verified because of the lack of any necessary plasma assessment. Finally, betaine may directly generate some saving of the SAMe rate found in the liquor, as was observed by some authors in patients affected by Alzheimer's disease. ${ }^{12}$ In this case, the administration of betaine would be tantamount to administering SAMe directly. Even in this case, the lack of plasma information and liquor analysis does not allow us to

Table 8 Compliance assessment

\begin{tabular}{llll}
\hline Compliance & N (SAMe) & N (SAMe/betaine) & $\boldsymbol{P}$ \\
\hline Poor & $\mathrm{I}$ & 0 & $\mathrm{~ns}$ \\
Fair & 10 & $\mathrm{I} 4$ & $\mathrm{~ns}$ \\
Good & 10 & 8 & $\mathrm{~ns}$ \\
Excellent & $\mathrm{I}$ & $\mathrm{I}$ & $\mathrm{ns}$ \\
\hline
\end{tabular}

Abbreviations: ns, not significant; SAMe, S-adenosyl-methionine. understand the mechanism of the data observed. This work obviously presents some limitations, and consequently, its results should be considered as only preliminary issues. The low number of individuals enrolled in the study and the lack of a placebo group or a double-blind trial do not allow us to provide a more definite representation of the issue. Another limitation may derive from the fact that some doses of antidepressants had not been increased prior to treatment with SAMe or the administration of SAMe plus betaine. For example, the patients treated with bupropion received a daily dose of $150 \mathrm{mg}$, and any additional effect was assessed on this dosage without first trying to increase the bupropion dose to $300 \mathrm{mg}$. This choice was obviously made to limit treatment toxicity. In addition, the comparison of two different doses of SAMe $(800 \mathrm{mg} /$ day in the SAMe group versus $750 \mathrm{mg}$ /day in the $\mathrm{SAMe} /$ betaine group) is partially flawed. The comparison of the doses could not be improved, however, because the drug is available on the market as 400-mg tablets, and to date, SAMe-based drugs associated with betaine in the same tablet are not available. Although nutraceutical preparations combining SAMe and betaine are available on the market, the dose of SAMe per tablet cannot exceed $250 \mathrm{mg}$ /day because of regulatory reasons concerning the world of nutraceuticals. In the authors' opinion, however, the comparison between 800 and $750 \mathrm{mg}$ is acceptable, at least in view of the demonstration they had planned to prove. The exceeding $50 \mathrm{mg}$ is to the advantage of the reference group rather than to the group where the authors hoped to be able to demonstrate a better clinical activity. So, the substance of the preliminary observation can still be maintained in spite of so many limitations: the administration of betaine and SAMe, when compared with the adjunctive treatment with SAMe alone, seems to improve the symptoms of patients affected by mild-to-moderate depression and poorly responsive to treatments performed with antidepressants other than SAMe. New double-blind trials versus placebo with equally dosed SAMe preparations, where plasma homocysteine levels will be analyzed and where the Beck Depression Inventory Scale II will be used to score the full length of the trial, are in progress to confirm the data put forward in this study.

\section{Conclusion}

On the basis of study results, it may be assumed that the administration of SAMe plus betaine is a better adjunctive therapy than the administration of SAMe alone in subjects with mild-to-moderate depression who are low responders to conventional antidepressive drugs. 


\section{Acknowledgment}

The authors thank Dr Paolo Risso for the statistical analysis of the results.

\section{Disclosure}

FDP is the main formulator of DDM Metile ${ }^{\circledR}$. The other authors report no conflict of interest.

\section{References}

1. Hardy ML, Coulter I, Morton SC, et al. S-adenosyl-L-methionine for treatment of depression, osteoarthritis, and liver disease. Evid Rep Technol Assess (Summ). 2003;64:1-3.

2. Mischoulon D, Price LH, Carpenter LL, et al. A double-blind, randomized, placebo-controlled clinical trial of S-adenosyl-L-methionine (SAMe) versus escitalopram in major depressive disorder. $J$ Clin Psychiatry. 2014;75(4):370-376.

3. Sarris J, Papakostas GI, Vitolo O, Fava M, Mischoulon D. S-adenosyl methionine (SAMe) versus escitalopram and placebo in major depression RCT: efficacy and effects of histamine and carnitine as moderators of response. J Affect Disord. 2014;164:76-81.

4. Spillmann M, Fava M. S-adenosyl-methionine (ademethionine) in psychiatric disorders. CNS Drugs. 1996;6:416-425.

5. Alpert JE, Mischoulon D. One-carbon metabolism and the treatment of depression: roles of S-adenosyl methionine (SAMe) and folic acid. In: Mischoulon D, Rosenbaum J, editors. Natural Medications for Psychiatric Disorders: Considering the Alternatives. Philadelphia: Lippincott Williams \& Wilkins; 2002:44.

6. Bertoia ML, Pai JK, Cooke JP, et al. Plasma homocysteine, dietary $\mathrm{B}$ vitamins, betaine, and choline and risk of peripheral artery disease. Atherosclerosis. 2014;235(1):94-101.

7. Permoda-Osip A, Dorszewska J, Skibinska M, Chlopocka-Wozniak M, Rybakowski JK. Hyperhomocysteinemia in bipolar depression: clinical and biochemical correlates. Neuropsychobiology. 2013;68(4): 193-196.

8. Narayan SK, Verman A, Kattimani S, Ananthanarayanan PH, Adithan C. Plasma homocysteine levels in depression and schizophrenia in South Indian Tamilian population. Indian J Psychiatry. 2014;56(1):46-53.

9. Owen RT. Folate augmentation of antidepressant response. Drugs Today (Barc). 2013;49(12):791-798.
10. Erbe S, Pellert UN. Folates in the treatment of depression. Fortschr Neurol Psychiatr. 2014;82(2):78-83.

11. Kharbanda KK, Rogers DD 2nd, Mailliard ME, et al. A comparison of the effects of betaine and S-adenosylmethionine on ethanol-induced changes in methionine metabolism and steatosis in rat hepatocytes. J Nutr. 2005;135(3):519-524.

12. Knopman D, Patterson M. An open-label, 24-week pilot study of the methyl donor betaine in Alzheimer disease patients. Alzheimer Dis Assoc Disord. 2001;15(3):162-165.

13. World Medical Association. World medical association declaration of Helsinki: ethical principles for medical research involving human subjects. J Postgrad Med. 2002;48(3):206-208.

14. Richter P, Werner J, Heerlein A, Kraus A, Sauer H. On the validity of the Beck depression inventory. A review. Psychopathology. 1998;31(3): 160-168.

15. Huskisson EC, Jones J, Scott PJ. Application of visual-analogue scales to the measurement of functional capacity. Rheumatol Rehabil. 1976;15(3):185-187.

16. Levkovitz Y, Alpert JE, Brintz CE, Mischoulon D, Papakostas GI. Effects of S-adenosylmethionine augmentation of serotonin-reuptake inhibitor antidepressants on cognitive symptoms of major depressive disorder. J Affect Disord. 2012;136(3):1174-1178.

17. Abularrage CJ, Sidawy AN, White PW, et al. Effect of folic acid and vitamins B6 and B12 on microcirculatory vasoreactivity in patients with hyperhomocysteinemia. Vasc Endovascular Surg. 2007;41(4): 339-345.

18. Olthof MR, Verhoef P. Effects of betaine intake on plasma homocysteine concentrations and consequences for health. Curr Drug Metab. 2005;6(1):15-22.

19. Schwab U, Törrönen A, Meririnne E, et al. Orally administered betaine has an acute and dose-dependent effect on serum betaine and plasma homocysteine concentrations in healthy humans. $J$ Nutr. 2006;136(1): 34-38

20. Olthof MR, van Vliet T, Boelsma E, Verhoef P. Low dose betaine supplementation leads to immediate and long term lowering of plasma homocysteine in healthy men and women. $J$ Nutr. 2003;133(12): 4135-4138.

21. Mischoulon D, Alpert JE, Arning E, Bottiglieri T, Fava M, Papakostas GI. Bioavailability of S-adenosyl methionine and impact on response in a randomized, double-blind, placebo-controlled trial in major depressive disorder. J Clin Psychiatry. 2012;73(6):843-848.
Journal of Multidisciplinary Healthcare

\section{Publish your work in this journal}

The Journal of Multidisciplinary Healthcare is an international, peerreviewed open-access journal that aims to represent and publish research in healthcare areas delivered by practitioners of different disciplines. This includes studies and reviews conducted by multidisciplinary teams as well as research which evaluates the results or conduct of such teams or

\section{Dovepress}

healthcare processes in general. The journal covers a wide range of areas and welcomes submissions from practitioners at all levels, from all over the world. The manuscript management system is completely online and includes a very quick and fair peer-review system. Visit http://www.dovepress.com/testimonials.php to read real quotes from published authors. 